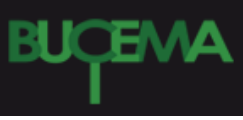

Bulletin du centre d'études médiévales d'Auxerre | BUCEMA

11 | 2007

Varia

\title{
Autour de Herbert Leon KESSLER
}

Auxerre, Centre d'études médiévales, 15-16 mars 2007

Daniel Russo, Herbert Leon Kessler, Dominique Donadieu-Rigaut, Dominique logna-Prat et Anne-Orange Poilpré

\section{(2) OpenEdition}

Journals

Édition électronique

URL : https://journals.openedition.org/cem/1381

DOI : 10.4000/cem.1381

ISSN : 1954-3093

Éditeur

Centre d'études médiévales Saint-Germain d'Auxerre

Édition imprimée

Date de publication : 15 août 2007

ISSN : 1623-5770

Référence électronique

Daniel Russo, Herbert Leon Kessler, Dominique Donadieu-Rigaut, Dominique logna-Prat et AnneOrange Poilpré, « Autour de Herbert Leon KESSLER », Bulletin du centre d'études médiévales d'Auxerre I BUCEMA [En ligne], 11 | 2007, mis en ligne le 30 août 2007, consulté le 22 septembre 2022. URL : http://journals.openedition.org/cem/1381; DOI : https://doi.org/10.4000/cem.1381

Ce document a été généré automatiquement le 22 septembre 2022.

\section{(c) (i) (2)(2)}

Creative Commons - Attribution - Pas d'Utilisation Commerciale - Partage dans les Mêmes Conditions 4.0 International - CC BY-NC-SA 4.0

https://creativecommons.org/licenses/by-nc-sa/4.0/ 


\title{
Autour de Herbert Leon KESSLER
}

\author{
Auxerre, Centre d'études médiévales, 15-16 mars 2007 \\ Daniel Russo, Herbert Leon Kessler, Dominique Donadieu-Rigaut, \\ Dominique logna-Prat et Anne-Orange Poilpré
}

1 En prenant pour point de départ l'ensemble de ses travaux, thèse, études plus précises sur les grandes périodes de l'histoire de l'art, et de l'art médiéval en particulier, essais théoriques, un groupe de chercheurs s'est réuni autour de Herbert Leon Kessler pour essayer de faire la synthèse, avec lui, de ses différents apports au champ des sciences humaines. Professeur à Johns Hopkins University, Baltimore (USA), Fellow de la Medieval Academy of America (1991) et de l'American Academy of Arts and Sciences (1995), Herbert L. Kessler a développé, et développe, ses recherches en suivant trois axes principaux de réflexion : la pertinence du comparatisme entre Orient byzantin et Occident latin pour l'art du Moyen Âge ; la notion et le statut d'image ; le concept d'art dans la perspective générale d'une histoire de l'art ouverte aux sciences sociales ${ }^{1}$.

Durant sa formation, et de son aveu personnel, Herbert L. Kessler a surtout conçu le goût et la nécessité de penser librement les objets qu'il étudiait. À l'Université de Princeton, il suivit les séminaires de Kurt Weitzmann (1904-1993) et d'Erwin Panofsky (1892-1968) qui, certes, orientèrent ses recherches, mais le persuadèrent de conserver toute sa liberté d'approche. Anne-Orange Poilpré y revient dans son analyse de l'œuvre de Herbert L. Kessler. Il en retira surtout deux principes qu'il s'efforça toujours de mettre en application. Le premier, à propos des 'images', revient à toujours les considérer sous leurs aspects matériels et, par conséquent, à ne plus employer le terme, rejoignant en cela les conceptions de certains artistes, tel Mark Rothko, à propos du 'processus organique de l'art'2. Le second, sur l'objet d'art et ses publics, est largement fondé sur la prise en compte de la notion d'audience, telle qu'elle a été formulée par Hans Belting dans ses travaux, notamment selon la perspective d'une anthropologie de l'art ${ }^{3}$ ouverte aux sciences sociales. Deux autres apports n'ont pas peu contribué à orienter ses recherches: les travaux sur l'art contemporain, d'abord, en particulier ceux d'Yves-Alain Bois sur Mondrian, mais aussi ceux d'autres critiques sur le mouvement expressionniste aux États-Unis; les réflexions toujours stimulantes de 
Michael Camille (1958-2002), ensuite, sur les franges d'un literary criticism pénétré de Gilles Deleuze, Jacques Derrida et Jean Baudrillard ${ }^{4}$.

3 En tenant compte de la richesse de ces approches croisées, Daniel Russo souligne comment Herbert L. Kessler renouvela l'étude des rapports entre les arts et la réforme de l'Église en s'intéressant à la question du narratif en peinture, aux modes de composition et aux emplacements dans les églises, à l'intérieur d'un espace territorialisé autour de Rome et dans Rome même, entre le $\mathrm{XI}^{e}$ et le $\mathrm{XIV}^{\mathrm{e}}$ siècle. Mesurant l'exemplarité des deux anciennes basiliques de Saint-Pierre de Rome et de Saint-Paul-hors-les-murs pour douze édifices situés dans l'espace latial, Herbert L. Kessler analysa, pour chacun d'entre eux et séquence après séquence, les choix iconographiques, leur distribution sur les murs, dans la nef ou dans le chœur, leurs relations avec les autres éléments du décor et, enfin, leur rôle actif dans la production d'un sens général qui passait, alors, plutôt par une suite d'échos dans les formes et les couleurs, les figures et les motifs, que par les reprises conceptuelles ou dogmatiques. Ainsi, à Cori, dans l'église de l'Annunziata, un petit oratoire au sud-est de Rome, daté de la fin du XIVe siècle pour les décors peints, l'élément mis en valeur fut le parallélisme délibéré entre les épisodes de l'ancien et ceux du nouveau Testament, avec une nette prédominance des premiers. Sur la Croix dite de Constantin, conservée aujourd'hui dans le Musée du Latran, à Rome, les épisodes des 'Histoires de Noé' entretinrent, à la fin du $\mathrm{XIV}^{\mathrm{e}}$ siècle, si la datation est vérifiée, un dense tissu de relations visuelles avec les grandes réalisations monumentales de la Rome des papes. À Ceri, dans l'église San Felice, le Jugement dernier peint au revers du mur de la façade tint à peu de chose près un rôle identique. Dans le prolongement de ces études minutieuses sur les traces et les empreintes du grand art romain, de l'antiquité chrétienne jusqu'aux XII ${ }^{e}$ et XIII ${ }^{e}$ siècles, deux a priori de la recherche en histoire de l'art comme en iconographie sont sérieusement remis en cause: la notion de renovatio puis celle, concomitante, de direction des arts sous le contrôle de la Papauté. Stefano Riccioni, Simone Piazza ont, chacun à sa manière, l'un explorant Rome, l'autre l'espace compris entre le sud du Latium et le nord de la Sabine, fait également justice de ces conceptions ${ }^{5}$.

4 En revenant sur l'apport d'Herbert L. Kessler à l'histoire de l'Église et à l'ecclésiologie médiévale, Dominique Iogna-Prat retrouve bien des éléments d'une typologie des représentations de l'ecclesia, au sens où l'entendait le père Yves Congar (1904-1995), et développe toute la question de l"économie du christianisme' ${ }^{6}$. Remarquant après Marie-José Mondzain que le mot 'économie' devient le leitmotiv appliqué à la défense iconique dans les traités de Nicéphore, Jean Damascène, Théodore Stoudite, il note son utilisation pour l'ensemble du plan incarnationnel, dans lequel on l'emploie, en particulier pour désigner tout le champ sémantique complexe régissant les rapports entre le sacré et le profane, le visible et l'invisible, le visible et le lisible, la rigueur de la loi et l'adaptabilité de la règle ${ }^{7}$. Car c'est bien le dogme de l'incarnation que vient soutenir ce terme en même temps qu'il sert à construire tout l'édifice de la pensée sur l'icône et sur la figurabilité de l'invisible. Par ce biais très éclairant, l'on rejoint certaines des affirmations de Herbert L. Kessler sur la question de la visibilité au Moyen Âge, même si le concept d'économie lui paraît trop organiciste et fonctionnaliste, éloigné donc de ce qu'il veut démontrer pour l'art de cette époque. Dans les faits, tels que Marie-José Mondzain les analyse à Byzance, par le concept d'économie, toute l'Église est identifiée au corps du Christ dont on doit pouvoir produire la visibilité afin 
que le royaume terrestre puisse se constituer à l'image du royaume céleste, dont il incarnera dès lors, 'ici-bas', la manifestation providentielle ${ }^{8}$.

Pour Anne-Orange Poilpré, parler de l'art chrétien antique dans l'œuvre de Herbert L. Kessler, c'est remonter aux origines, mais aussi aller à quelque chose d'essentiel. En effet, son intérêt pour l'Antiquité chrétienne réside au fondement même de sa formation universitaire puisqu'il fut l'élève de Kurt Weitzmann. Sous sa direction, il mène à bien un travail de thèse sur les frontispices de la Genèse des Bibles de Charles le Chauve, qu'il soutient à Princeton en 1965, et publie dans l'Art Bulletin en $1971{ }^{9}$. Par son apprentissage et des années passées auprès de lui, Herbert L. Kessler a été profondément marqué. Bien après la retraite de Kurt Weitzman, professeur à l'Université de Princeton jusqu'en 1972, et après avoir entamé une carrière internationale à l'Université de Chicago, puis à Baltimore (Johns Hopkins), Herbert L. Kessler poursuit une réflexion commune et les collaborations avec son ancien maître.

6 En 1986, il collabore à l'ouvrage de Weitzmann sur la Genèse de Cotton et coécrit avec lui en 1990 la somme sur les peintures de la Synagogue de Doura Europos (1990). Parmi les thématiques artistiques et les méthodologies explorées par Kurt Weitzmann, plusieurs jouent un rôle déterminant dans la démarche de Herbert L. Kessler. Le sujet de sa thèse s'élabore grâce aux suggestions de Weitzmann dont les recherches sur les Bibles tardo-antiques, et en particulier sur l'illustration de la Septante, mettent en évidence la nécessité d'un travail réactualisé sur le corpus iconographique carolingien. Pour cela, Weitzmann met à sa disposition toute sa documentation personnelle, en particulier celle qui, alors, est encore inédite sur la Genèse de Cotton.

C'est d'ailleurs comme un réexamen de l'étude de Wilhelm Köhler («a reassessment » selon l'efficace expression anglophone) d'après laquelle les manuscrits de Tours, en particulier la Bible de Saint-Paul-hors-les-murs, seraient copiés sur des manuscritsmodèles $\mathrm{du} \mathrm{V}^{\mathrm{e}}$ siècle, qu'est amenée la publication du livre issu de son $\mathrm{PhD}^{10}$. D'après Köhler, les préoccupations artistiques et religieuses révélées par les thèmes iconographiques de la Bible de Saint-Paul seraient si étrangères au IX ${ }^{e}$ siècle, mais au contraire si proches de celles du milieu du $\mathrm{V}^{\mathrm{e}}$ siècle et du pontificat de Léon le Grand, que seul le truchement d'un modèle paléochrétien (formé d'une bible complète) saurait expliquer l'originalité de ces œuvres.

Deux aspects majeurs à cette démarche. L'un méthodologique est de démontrer ainsi la force de l'analyse iconographique contre le type d'organisation de corpus choisi par Köhler, qui raisonne par école stylistique. Pour cela, les méthodes élaborées par Kurt Weitzmann dans Illustrations in Roll and Codex ${ }^{11}$ (paru pour la première fois en 1947) vont offrir à son travail un cadre renouvelé et dynamique. En effet, l'intérêt de Weitzman pour l'illustration des livres de la Bible depuis les origines, de la Septante en particulier, donnant au corpus iconographique grec une visibilité qu'on lui accordait peu, permet une profondeur inédite dans l'établissement d'une chronologie et dans la recherche des modèles.

9 Le deuxième aspect majeur de cette démarche est d'insister sur une certaine vision de l'Antiquité chrétienne. En effet, depuis les années 1930 qui ont vu paraître les premiers travaux de Wilhelm Köhler, la connaissance des manuscrits chrétiens antiques s'est considérablement améliorée. La connaissance de l'héritage artistique, de l'aspect de modèles aujourd'hui perdus mais perceptibles grâce à des œuvres plus tardives ellesmêmes tournées vers l'Antiquité chrétienne, permet de dresser une sorte de portrait de ces traditions illustratives anciennes. 
10 La connaissance de manuscrits grecs tels que la Genèse de Cotton va, bien sûr, se révéler d'une pertinence directe et capitale, quant à l'étude et à la compréhension des frontispices de la Genèse dans le corpus du IX ${ }^{e}$ siècle, levant un coin du voile sur un continent perdu. D'ailleurs, dès l'introduction et l'état de la question, Herbert L. Kessler souligne bien à quel point les travaux de Weitzmann sur l'illustration des différents livres de l'Ancien Testament (comme la Genèse et les Psaumes), ou bien encore celle des Actes de Apôtres, ont changé les choses et conditionné sa méthode.

$11 \mathrm{Au}$ cours de ces années propédeutiques, l'enthousiasme de Herbert L. Kessler, qu'orientent l'expérience et l'élan des recherches de Kurt Weitzmann, va donner à l'Antiquité une place centrale, tant dans ses démarches iconographiques que conceptuelles. Interroger le pourquoi et le comment de la représentation chrétienne, penser les médiations sensibles et symboliques par lesquelles on représente l'invisible dans l'art chrétien, passe souvent par un retour vers les phénomènes artistiques de l'Antiquité en tant qu'ils nourrissent aussi de nombreuses œuvres médiévales. On peut réitérer l'exemple des Bibles carolingiennes. Cela vaut également pour des travaux concernant l'iconographie des apôtres, en particulier Pierre et Paul. Une Antiquité chrétienne finalement pensée et vécue comme omniprésente dans le Moyen Âge, source d'inspiration, et âge d'or, temps des origines rêvées et réinventées, parfois par les historiens de l'art, mais aussi par les acteurs eux-mêmes. S'affirme ici une démarche adhérant probablement au plus juste de la complexité, la subtilité, et parfois la volatilité, des processus iconographiques et artistiques dans le Christianisme.

12 En 1990, l'étude à quatre mains sur les Fresques de Doura Europos intitulée: "Les Fresques de la synagogue de Doura et l'art chrétien ${ }^{12}$, dresse un portrait précis de ce qu'est précisément l'Antiquité où naît l'art chrétien. Pour Herbert L. Kessler et Kurt Weitzmann, il s'agit d'une Antiquité certes païenne, mais surtout imprégnée d'une culture, tant littéraire qu'iconographique, juive et judéo-chrétienne. Dans la partie qu'il rédige, Weitzmann reformule des thématiques et des conclusions présentes dans ses travaux depuis les années 1940 (et la parution de Illustrations in Roll and Codex). Son idée est moins d'expliquer les peintures de la synagogue en tant que telles mais de comprendre comment elles peuvent nourrir la reconstitution d'une illustration des livres de la Bible. Autour de cette œuvre exceptionnelle, il entreprend donc de reconstituer un stemma, de penser des procédés de transmissions restituant le contexte artistique et culturel, interrogeant par là l'idée d'une œuvre hapax à Doura.

13 C'est surtout l'illustration de livres juifs anciens, bibliques et littéraires, qu'attestent les peintures de Doura Europos. Weitzmann pense surtout à la version grecque de la Bible, en usage dans les milieux hellénisés juifs comme chrétiens. D'ailleurs - et c'est là un aspect important de ses conclusions - Weitzmann souligne bien la communauté de modèles d'illustrations bibliques pour les juifs et les chrétiens, dans les deux ou trois premiers siècles de notre ère.

14 Ainsi déboute-t-il la question de la priorité donnée à l'illustration «chrétienne » ou «juive » de l'Ancien Testament puisque sa préoccupation est moins chronologique que thématique, celle d'une recension des images elles-mêmes et de leur relation à un prototype, quel qu'il soit. C'est l'un des aspects majeurs des thèses de Weitzmann de penser que, au moins dans le contexte syrien de Doura, les communautés chrétiennes et juives ont échangé et partagé leurs livres en grec. Les livres bibliques ont possédé une ornementation développée au milieu du $\mathrm{III}^{\mathrm{e}}$ siècle, date du cycle de la synagogue de Doura, c'est tout ce qu'il est raisonnable d'avancer en matière de chronologie. 
15 Autre questionnement : l'existence d'une tradition iconographique narrative dans l'art juif et chrétien, qui touche à une question fondamentale quant à la naissance de l'art chrétien. Weitzmann réitère la trame même de son approche depuis Roll and codex qui situe la naissance des procédés narratifs iconographiques utilisés dans l'art chrétien non pas dans l'art chrétien lui-même, mais plus lointainement dans l'art hellénistique. En effet, les procédés capables d'organiser des cycles narratifs élaborés au IV ${ }^{\mathrm{e}}$ siècle avant J.-C. sont précisément ceux dont héritent la figuration juive comme la figuration chrétienne.

16 Ces thèses modèlent, en vérité, toute une façon de concevoir les origines de l'art chrétien dans l'Antiquité. L'iconographie qui s'élabore pendant les quatre premiers siècles du christianisme, mal connue par les œuvres, est à la fois l'objet de spéculation mais aussi le support d'une certaine idée sur la façon dont le christianisme s'est imposé dans le monde méditerranéen: deux alternatives différentes peuvent tracer le cheminement vers une réponse: un art chrétien nourri et façonné par l'univers culturel préexistant (païen, latin, hellénique, juif) ou bien doué d'une formidable capacité d'invention de son propre récit iconographique dès lors qu'il est entériné par la sphère politique.

17 Des travaux comme ceux d'André Grabar (1886-1990), savant de la même génération que Kurt Weitzmann, professent un point de vue différent. Son approche de l'Antiquité tardive et de l'art chrétien - qui jusqu'à aujourd'hui conditionne bon nombre de perspectives sur ces questions en France - donne à ce qu'il appelle les « images-signes » une antériorité chronologique par rapport aux images proprement narratives ${ }^{13}$. Pourtant, il reconnaît bien l'originalité des Genèse de Cotton et de Vienne, et envisage la possibilité d'un modèle juif, mais pas au point d'envisager le fait que l'art chrétien manie déjà des procédés narratifs et cycliques en certaines occasions, séparant ainsi très nettement ce qui relève de l'Ancien Testament et du Nouveau.

18 Le point de vue de Weitzmann traduit plutôt une perception de l'Antiquité où se mêlent et se partagent des influences tant romaines que païennes, hellénistiques, juives, dans l'élaboration de la narration imagée chrétienne. Et cela conditionne la perception même de cette religion et de son rapport à la figuration sacrée.

19 La partie rédigée par Herbert L. Kessler dans l'ouvrage sur Doura est justement pensée en complémentarité de ces thèses de Weitzmann. En réfléchissant sur les dimensions programmatiques des peintures de la synagogue, Herbert L. Kessler propose des comparaisons avec des ensembles ornementaux paléochrétiens plus tardifs de façon à illustrer cette idée d'un héritage de la narration imagée juive. Pour lui, plus encore que les édifices chrétien, la synagogue semble détenir les clés d'une explication sur la formation de l'imagerie chrétienne. Il souligne par exemple les parentés existant entre certains modes d'organisation des images autour d'un point focal de l'édifice sacré. La présence des figures prophétiques telles que celles de Moïse, Isaïe, ou Jérémie, est relevée tant dans la partie centrale de la synagogue de Doura que dans le chœur des édifices chrétiens comme Saint-Vital à Ravenne. L'insistance sur certains objets rituels, les références symboliques appuyées au Temple de Jérusalem, évoquent également certaines iconographies chrétiennes telles que le trône vide entouré des Vivants au sommet de l'arc de chœur dans Sainte-Marie-Majeure, à Rome, au V $\mathrm{V}^{\mathrm{e}}$ siècle.

Par ailleurs, quant au cycle de Doura en lui-même, Herbert L. Kessler montre la pertinence symbolique de l'ensemble quant aux polémiques religieuses et dogmatiques animant les communautés juives et chrétiennes vers le milieu du $\mathrm{III}^{\mathrm{e}}$ siècle. En effet, 
lorsqu'est construite une synagogue à Doura, petite ville accueillant donc une communauté juive et une communauté chrétienne, les polémiques entre elles se concentrent sur trois points principaux: le Christ a-t-il accompli les prophéties messianiques de l'Ancien Testament, en étant donc lui-même le Messie ? La destruction du Temple de Jérusalem a-t-elle constitué une rupture dans l'Alliance de Dieu et du peuple Hébreu ? L'Ancien Testament doit-il être interprété et compris littéralement ou typologiquement? Ainsi, des passages critiques de l'Ancien Testament dans cette polémique (le sacrifice d'Isaac, la bénédiction de Jacob, les prophéties concernant l'ascendance davidique du Messie) sont précisément centraux dans le décor de Doura. D'un côté invoqués en faveur d'une lecture littérale de l'Ancien Testament, parole de Dieu, de l'autre pour illustrer la nécessité d'une lecture christologique. Ainsi les citations « littérales» du texte biblique dans le décor de la synagogue de Doura sontelles, en elles-mêmes des arguments en faveur de l'interprétation juive. Aspect majeur du travail iconographique de Herbert L. Kessler sur l'Antiquité chrétienne, l'image, en tant que telle, est un argument apporté au débat théologique. Seule une iconographie narrative fondée sur la dimension historique des textes, réunis dans la salle en une même démonstration, était à même d'appuyer la force de l'argument historique et littéral contre la perspective chrétienne.

21 En 1986, paraît enfin la somme sur la Genèse de Cotton, mûrie pendant des décennies ${ }^{14}$. Il s'agit à nouveau d'un livre à quatre mains (avec Kurt Weitzmann), mais où cette fois les apports de chacun se confondent en un même texte. Avec cette parution accède enfin aux yeux du public une somme fondamentale sur les origines de l'illustration biblique chrétienne à travers une présentation exhaustive et critique de l'un des plus anciens cycles bibliques illustrés. Il s'agit également d'un pivot dans la réflexion menée par Kurt Weitzmann et Herbert L. Kessler depuis de très nombreuses années.

Le corps même de l'étude propose une analyse rigoureuse de chacun des fragments restants, la reconstitution complète de l'aspect général du manuscrit et de la disposition des cadres réservant les zones peintes, ainsi qu'une recherche des sources littéraires, iconographiques et des modèles possibles (selon la méthode élaborée par Weitzmann) : tout cela permet aux auteurs de restituer tout un paysage religieux et artistique contemporain de la réalisation du manuscrit. Cette Genèse chrétienne, datée $\mathrm{du} \mathrm{V}^{\mathrm{e}}$ siècle, marquée par un vocabulaire figuré chrétien déjà bien développé, aurait ainsi pour modèle un prototype des environs de 200. Cette étude développe une vision spécifique de l'art de Antiquité chrétienne - dont les publications antérieures de Weitzmann et de Herbert L. Kessler avaient donné la mesure - portée cette fois par l'une de ses œuvres majeures. Il s'agit d'une vision que l'on pourrait qualifier d'«ouverte». Ouverte vers le passé puisque c'est bien à l'aune des procédés narratifs élaborés dans l'Antiquité païenne, entre autres dans les milieux juifs hellénisés que se construit la narration biblique chrétienne, nourrie des échanges, des controverses de l'extraordinaire dynamisme intellectuel et artistique des premiers siècles de notre ère en Méditerranée. Ouverte vers le futur car il s'agit bien ici des modèles iconographiques des Bibles complètes réalisées à partir de l'époque carolingienne et du règne de Charles le Chauve. Par ailleurs, les auteurs montrent également comme sont nombreux les emprunts à d'autres formes artistiques que l'illustration de textes: art monumental paléochrétien, sculpture sur ivoire, peintures et sculptures antiques. 
rupture fondamentale par rapport au carcan des catégories techniques et géographiques pour l'étude artistique, sous son versant iconographique en particulier, caractérise également de nombreux travaux d'Herbert L. Kessler sur l'art chrétien antique. Dans le sillage de l'exceptionnelle exposition Age of Spirituality, organisée en 1977-1978 par Kurt Weitzmann et Margaret Frazer au Metropolitan Museum of Art, Herbert L. Kessler revient, après la parution de son livre sur les Bibles tourangelles en 1977, sur des questions d'iconographie paléochrétienne. Des publications telles que "Scenes from the Acts of the Apostles on Some Early Christian Ivories ", dans Gesta en $1979^{15}$, « Passover in St Peters ", dans Jewish Art en 1986/87 ${ }^{16}$, « The Meeting of Peter and Paul in Rome", dans les Dumbarton Oaks Papers en $1987^{17}$, ou bien "Caput et speculum omnium Ecclesiarum » en $1989^{18}$, supportent une réflexion continue sur le caractère essentiel des phénomènes iconographiques tardo-antiques: comme constitutifs d'une iconographie chrétienne en pleine construction, mais aussi comme les références et les modèles d'un christianisme médiéval plus tardif en perpétuelle référence à son passé et à ses origines apostoliques.

Dominique Donadieu-Rigaut revient sur l'article intitulé "Passover in St Peter's", paru pour la première fois en 1987 dans la revue Jewish Art, dans lequel Herbert L. Kessler se livre à l'analyse d'un cycle de fresques disparu, celui de la "première " basilique Saint-Pierre érigée au $\mathrm{IV}^{\mathrm{e}}$ siècle sous l'impulsion de Constantin, puis détruite pour laisser place à l'édifice baroque que nous connaissons tous.

Cette étude pourrait paraître paradoxale au sein d'une œuvre consacrée en grande partie à la question du regard au Moyen Âge, comme en témoigne plus que jamais le dernier ouvrage d'Herbert L. Kessler, Seeing medieval art (2004).

En effet, comment l'historien de l'art, pour qui l'observation directe, le face à face avec l'original, constitue la condition sine qua non de l'analyse des œuvres, peut-il bien procéder pour étudier des images désormais invisibles, volontairement détruites depuis des siècles?

C'est tout le défi méthodologique que relève Herbert L. Kessler dans cet article, développé par quelques-unes de ses autres contributions majeures ${ }^{19}$. Puisque l'œuvre d'un chercheur se mesure aussi à sa propension à susciter des échos chez les autres, des pistes, des interrogations, Dominique Donadieu-Rigaut prolonge (comme en un fil tiré), l'une des observations d'Herbert L. Kessler, tout à fait intrigante. Comme nous venons de le voir, en effet, l'auteur remarque que le passage de la Mer Rouge, à Cori, occupe deux séquences contiguës, situées en fin de cycle, juste avant l'invitation faite aux Élus de pénétrer au Paradis.

Alors que toutes les séquences narratives de ce cycle sont séparées les unes des autres par des colonnes fictives, peintes sur le mur, qui rythment et structurent l'ensemble du décor, le passage de la Mer Rouge occupe un double espace unifié, sans colonne centrale. On perçoit néanmoins, dans la composition même de cette large séquence, le souvenir d'une frontière verticale puisque la rive opposée, celle où se tiennent maintenant Moïse et les Fils d'Israël, dessine une ligne venant prolonger, avec un petit décalage, la colonne fictive qui se trouve au registre supérieur.

Cette particularité se retrouve dans une autre église italienne, la collégiale de San Gimignano (en Toscane, donc relativement loin de Rome). Dans cette église, il est difficile d'établir des correspondances précises et signifiantes entre le cycle de l'Ancien Testament (dans le bas-côté gauche en entrant) et celui du Nouveau Testament qui lui

Bulletin du centre d'études médiévales d'Auxerre | BUCEMA, 11 | 2007 
fait face (dans le bas-côté droit), car ils résultent de deux campagnes de décoration différentes, réalisées au XIV ${ }^{e}$ siècle par deux peintres différents. D'autre part, les sens de lecture de ces deux programmes s'avèrent très peu compatibles, voire divergents, d'un côté à l'autre de l'édifice.

Toujours est-il que le cycle vétérotestamentaire (constituant une unité picturale indéniable) présente une " anomalie » riche de sens au sein de la composition générale.

Les scènes de l'Ancien Testament sont disposées sur trois registres, qui se lisent tous les trois dans le même sens, depuis la porte d'entrée jusqu'au fond de l'église. Elles débutent en haut, au niveau des lunettes, avec la Création, pour se terminer au registre inférieur, au niveau de la $6^{\text {ème }}$ travée, par l'Histoire de Job (la 7 ème travée a été endommagée par le percement d'une chapelle). Au sein de cet agencement régulier, les séquences de ce cycle se trouvent bien séparées les unes des autres par tout un système de bordures décoratives reprenant les articulations architecturales de l'édifice, à savoir les travées. Le passage de la Mer Rouge, encore une fois, fait figure d'exception. Comme à Cori, il occupe l'espace de deux panneaux mitoyens dont la séparation verticale a été supprimée.

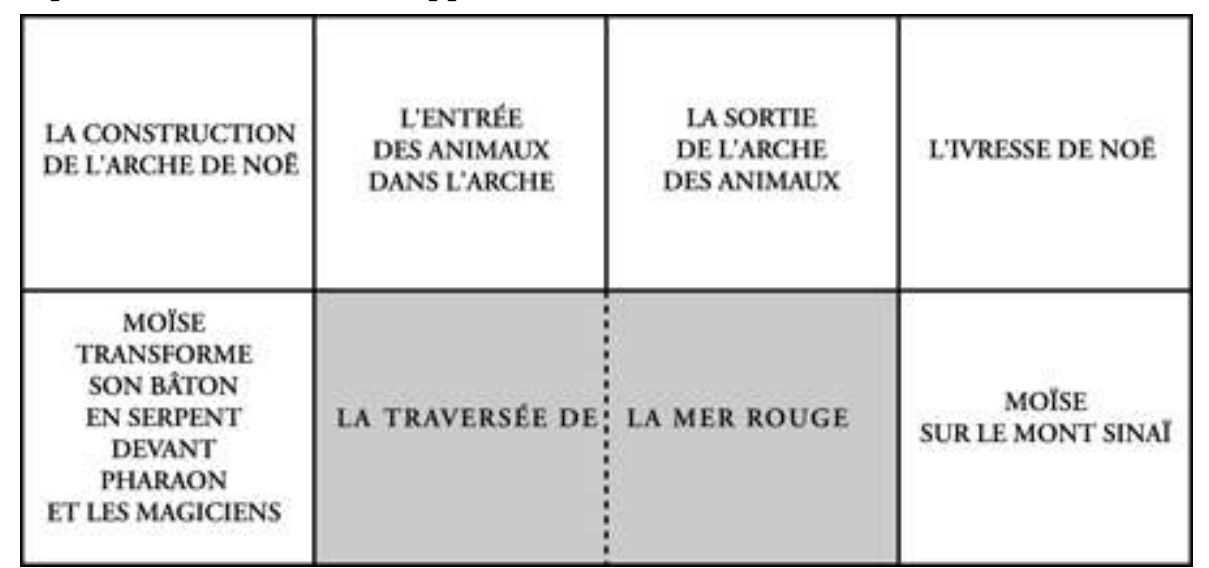

Bartolo di Fredi, Scènes de l'Ancien Testament (détail), 1367. Collégiale de San Gimignano, bas-côté gauche.

Cette rupture au sein de la scansion du cycle (à laquelle l'œil s'était habitué), relève, pour reprendre un terme de Louis Marin, de la "syncope", de l'introduction d'un élément hétérogène dans une unité figurative cohérente, faisant un signe fort au spectateur. C'est en effet cette absence de limite là où l'on attendait une frontière, une résistance visuelle, qui produit le miracle en image. Aucun obstacle ne s'est élevé devant les Hébreux, aucune barrière n'est venue entraver leur passage. Ils sont déjà de l'autre côté, hommes, femmes et enfants. Moïse, quant à lui, contemple l'action divine menée en la faveur de son camp. Deux éléments, l'un interne à l'image, l'autre relevant de sa situation dans l'édifice, entretiennent néanmoins le souvenir du cadre, ou plutôt le sentiment de son absence.

La lisière du rivage, étonnamment fine et rectiligne, réinstaure, non pas une limite, mais un partage, une distinction entre les bons et les méchants, entre les sauvés et les noyés, à l'intérieur même de cette double image unifiée. D'autre part, l'emplacement même de ce double espace ne coïncide pas avec une travée bien précise mais se trouve au contraire « à cheval » entre la $2^{\text {ème }}$ et la $3^{\text {ème }}$ travée. L'idée de passage joue aussi de ce décalage par rapport aux articulations architecturales de l'édifice. 
Plutôt que d'analyser l'omission du cadre entre les deux volets du miracle comme relevant d'une simple tradition iconographique, se répétant de site en site, comme sans y penser, je propose d'y voir un travail de la " pensée figurative " (Pierre Francastel) sur la notion de passage, de frontière, focalisé sur un épisode fondamental de la Bible incarnant précisément l'une des charnières essentielles de l'Histoire du Salut.

En effet, si l'on considère maintenant les deux scènes vétérotestamentaires situées juste au-dessus du passage de la Mer Rouge à San Gimignano, on est frappé par une autre «omission » qui affecte, cette fois-ci, la continuité narrative elle-même. À gauche est représentée l'entrée des animaux dans l'arche de Noé, puis immédiatement à droite leur sortie. De façon incroyable, le Déluge, événement central de la vie de Noé (et de l'humanité pécheresse), a été totalement nié, gommé.

3 Le « vrai » déluge, celui qui extermine la mauvaise engeance, se situe en fait au registre inférieur. La disposition très habile des scènes les unes au-dessus des autres invite donc le regard, à cet endroit précis, à opérer un décrochage dans sa lecture régulière et horizontale. Ce faisant, le passage de la Mer Rouge gagne en densité biblique : il demeure bien sûr, dans la logique de son registre, l'épisode fondamental de l'Exode marquant le passage de l'esclavage à la libération, mais il devient aussi la représentation paradigmatique de la purification par l'eau dont le déluge est le premier avatar.

7 Au-delà du miracle de l'Exode, le passage de la Mer Rouge cristallise ainsi cette idée de 'gond' dans l'histoire du Salut, de transition entre l'état d'esclavage et celui de libération, entre l'état de péché et celui de purification. Un passage qui fonctionne par l'utilisation symbolique de l'eau mise en scène dans les images mais aussi au cours d'un rituel de renaissance, le baptême. La collégiale de San Gimignano est en effet une pieve, c'est-à-dire une église paroissiale pourvue de fonts baptismaux. Les représentations murales trouvent ainsi leur résonance et leur actualisation dans la pratique sacramentelle se déroulant à l'intérieur de l'édifice.

8 Ce travail des images sur la notion de passage liée à l'épisode miraculeux de la Mer Rouge n'est pas l'apanage des peintures murales. On le retrouve également dans les manuscrits.

Le fait est particulièrement flagrant dans le célèbre Psautier de Canterbury. Le feuillet qui nous intéresse ici ( $\left.{ }^{\circ} 2\right)$ fut réalisé en Angleterre vers 1200.

Cette pleine-page relate différents épisodes de l'Exode centrés sur l'histoire de Moïse, depuis le mariage de ses parents jusqu'au miracle du rocher de Mara et Mériba.

Au sein de cette image composée de douze séquences rectangulaires, bien ordonnées entre elles par trois des quatre registres, le passage de la Mer Rouge occupe encore une fois une place singulière dans la mesure où deux séquences juxtaposées lui sont consacrées. L'eau de la mer, verte et translucide, forme une véritable montagne aquatique qui se répand dans la seconde vignette, malgré la séparation géométrique entre les deux séquences. Le fin bâton de Moïse, opérateur du miracle, fait office de trait d'union entre les deux vignettes. Le caractère quasiment maléfique des Égyptiens (figures du péché), est souligné par des diablotins qui s'agitent au-dessus des flots. À droite, sous les pieds des Hébreux, bien au sec entre deux replis de terrain, la mer poissonneuse a déjà retrouvé toute sa sérénité. L'ensemble du peuple élu se retourne alors pour constater visuellement la puissante intervention de Yahvé en sa faveur. 
42 Si le cadre est ici maintenu, on assiste néanmoins à sa transgression, ou plutôt à la négation de sa fonction puisque l'eau, comme le bâton efficient de Moïse, passe outre cette frontière, allègrement.

43 Parmi les multiples modalités figuratives se jouant de la limite, du cadre, de la frontière, Dominique Donadieu-Rigaut termine par un autre exemple, tiré de la Bible de Maciejowski enluminée en France du Nord vers 1250.

L'enluminure occupe la quasi-totalité du feuillet 9. Elle est construite sur deux registres eux-mêmes subdivisés en leur milieu par une colonne partie prenante d'un cadre architecturé. Ces deux colonnes au fût noir départagent les fonds colorés des registres, en créant un effet de chiasme entre les fonds d'or d'une part, les fonds bleu et rouge d'autre part. Si l'on observe attentivement ces deux colonnes, qui pourtant se ressemblent, il apparaît clairement qu'elles n'assument plus du tout, par rapport au récit, la même fonction. En bas, la colonne instaure une limite entre deux épisodes, reprenant ainsi la structure narrative de la Bible: une fois la Mer Rouge franchie, Myriam, sœur d'Aaron, entame, avec ses compagnes, un hymne en l'honneur de Yahvé, qui se manifeste par des chants, de la musique, et des danses. À la droite de cette séquence est figuré l'épisode qui lui fait immédiatement suite dans le texte : l'eau de Mara. Après trois jours de marche dans le désert, les Hébreux, assoiffés, tentent de s'abreuver à la source de Mara. Les eaux étant amères et imbuvables, Moïse jette un morceau d'écorce dans la source qui devient immédiatement potable (encore une histoire d'eau).

45 Au registre supérieur, au contraire, si la colonne institue une limite, c'est pour donner plus de force à sa transgression.

46 Est dès lors bien visible un double « débordement de la bordure ", derrière et devant la colonne: d'une part, l'eau de la Mer Rouge, qui n'a pas de couleur propre, semble tout à coup conférer au fond bleu de l'image, qui se présente comme un aplat, une texture singulière. Comme si la mer, en débordant, malaxait en quelque sorte le fond de l'image, le re-travaillait dans le sens d'une matérialité qu'il n'avait pas au départ, par une opération qui tient à la fois de la «dilution» et de la «coagulation ». D'autre part, les chevaux de Pharaon, sa couronne dorée, et un de ses soldats, culbutent par devant la colonne, bousculant ainsi non seulement la structure de l'image en deux séquences consécutives mais aussi l'épaisseur de l'image, puisqu'un étrange feuilletage des plans (pour reprendre ce terme à Jean-Claude Bonne), apparaît précisément à ce niveau.

47 Au total, il s'agit peut-être moins, de nos jours, pour l'historien de l'art du Moyen Âge, d'analyser l'évolution des formes que de donner du sens aux répétitions, aux duplications, aux "bégaiements» des images, sans pour autant faire fi des "petits écarts ", souvent très signifiants. On ne peut certainement pas, à cet égard, élaborer une théorie unique, justifiant de la même façon tous ces phénomènes. Certains relèvent de l'impact d'un modèle prégnant, érigé en référence absolue par un pouvoir politique qui construit, dans la longue durée, un réseau ecclésial. D’autres concernent plutôt la mise en image de la notion de passage, cristallisée sur un épisode biblique qui vaut finalement pour tous les rituels de purification par l'eau, dans la perspective d'un temps chrétien téléologique. Dès lors, dans quelle mesure peut-on encore parler de « tradition iconographique »? 


\section{NOTES}

1. Parmi les nombreux travaux d'H. L. KESSLER, nous citerons : The Illustrated Bibles from Tours, Princeton University Press, 1977 (Studies in Manuscript Illumination, 7) ; Studies in Pictorial Narrative, Londres, 1994 ; The Poetry and Painting of the First Bible of Charles the Bald, en collaboration avec P.E. Dutton, University of Michigan Press, 1997 ; Rome 1300 : On the Path of the Pilgrim, en collaboration avec J. Zacharias, Yale University Press, 2000 ; Spiritual Seing. Picturing God's Invisibility in Medieval Art, Philadelphie, The University of Pennsylvania Press, 2000 ; The Frescoes of the Dura Synagogue and Christian Art, en collaboration avec K. Weitzmann, Washington, 1990 (2003) ; Seeing Medieval Art, Petersborough (Ont.), 2004 (trad. fr. en cours aux éditions Macula).

2. M. Rотнко, The Artist's Reality. Philosophy on Art, New Haven/Londres, 2004, en part. chap. 3, (trad. fr. par P.-E. DAUZAT, Paris, 2004).

3. H. BELTING, Bild-Anthropologie : Eintwürfe für eine Bildwissenschaft, Munich, 2001 (trad. fr. par J. TORRENT, Paris, 2004).

4. M. CAMILlE, Image on the Edge: The Margins of Medieval Art, Cambridge, 1992 (trad. fr. par B. et J.-C. B , Paris, 1997) ; ID., Master of Death. The Lifeless Art of Pierre Remiet, Illuminator, New Haven/Londres, 1996.

5. S. Riccioni, Il mosaico absidale di San Clemente a Roma. Exemplum della Chiesa riformata, Spolète, Centro di Studi sull'alto Medioevo, 2006 ; S. PIAZZA, Pittura rupestre medievale. Lazio e Campania settentrionale (secoli VI-XIII), Rome, 2007.

6. Y. Congar, o. p., L'Église. De saint Augustin à l'époque moderne, 'Histoire des dogmes', t. 3, Paris, 1970 ; Cardinal Yves Congar, 1904-1995, (Rome, 3-4 juin 1996), A. VAUCHEZ (dir.), Paris, 1999. D. IogNA-PRAT, La Maison-Dieu. Une histoire monumentale de l'Église au Moyen Âge, Paris, 2006. 
7. M.-J. MonDZAIN, « Visage du Christ, forme de l'Église », in Du visage, textes réunis par M.-J. BAUDINET, Ch. SCHLATTER, Lille, 1982 ; EAD., Image, icône, économie. Les sources byzantines de l'imaginaire contemporain, Paris, 1996, en part. p. 95-210.

8. Pour une réflexion d'ensemble, J. ASSMANN, Le prix du monothéisme, (trad. de l'all. par L. BERNARDI), Paris, 2007, en part. p. 95-139 (« Le combat des souvenirs : idolâtrie et iconoclasme »).

9. H. L. KESSLER, « Hic homo formatur : The Genesis Frontispieces of the Carolingian Bibles », in The Art Bulletin, 53/2 (1971), p. 143-160.

10. H. L. KESSLER, The Illustrated Bibles from Tours, Princeton, 1977.

11. K. WeItZmanN, Illustrations in Roll and Codex. A Study of the Origin and Method of Text Illustration, Princeton, 1947.

12. K. WeITZMANN, H. L. KeSSLER, The Frescoes of the Dura Synagogue and Christian Art, Washington (Dumbarton Oaks), 1990.

13. A. GRABAR, Les Voies de la Création en iconographie chrétienne, Paris, 1979, chap. « La scène historique ", p. 85, 88, 91. Sur la portée des travaux d'André Grabar, M.-G. MUZJ, Un maître pour l'art Chrétien, André Grabar. Iconographie et théophanie, Paris, 2005.

14. K. WeItZMAnN, H. L. KessLer, The Cotton Genesis (British Library Codex Cotton Otho B. VI), Princeton, 1986.

15. H. L. KESSLER, « Scenes from the Acts of the Apostles on Some Early Christian ivories », in Gesta, 18/1 (1979), p. 109-119.

16. H. L. KeSSLER, « Passover in St Peters », in Jewish Art, 12-13 (1986-1987), p. 168-178.

17. H. L. KESSLER, « The meeting of Peter and Paul in Rome : an emblematic narrative of spiritual brotherhood », in Dumbarton Oaks Papers, 41 (1987), p. 265-275.

18. H. L. KESSLER, « Caput et speculum omnium Ecclesiarum » : Old Saint-Peter's and Church Decoration in Medieval Latium », in W. TRonzo (éd.), Italian Church Decoration of Middle Ages and Early Renaissance. Functions, Forms and Regional Traditions, Bologne, 1989, p. 119-146.

19. H. L. KESSLER, Old Saint Peter's and Church Decoration in Medieval Italy, Spolète, 2002.

\section{INDEX}

Mots-clés : Kessler Herbert Leon 